\title{
Universal algebra for general aggregation theory: Many-valued propositional-attitude aggregators as MV-homomorphisms*
}

\author{
Frederik Herzberg ${ }^{\ddagger}$ \\ Copyright notice: The final publication, in the Journal of Logic and Computation 25 \\ (2015), no. 3, pp. 965-977, is available at academic.oup.com, \\ doi:10.1093/logcom/ext009
}

\begin{abstract}
This paper continues Dietrich and List's [2011] work on propositionalattitude aggregation theory, which is a generalised unification of the judgement-aggregation and probabilistic opinion-pooling literatures. We first propose an algebraic framework for an analysis of (many-valued) propositional-attitude aggregation problems. Then we shall show that systematic propositional-attitude aggregators can be viewed as homomorphisms - algebraically structure-preserving maps — in the category of C.C. Chang's [1958] MV-algebras. (Proof idea: Systematic aggregators are induced by maps satisfying certain functional equations, which in turn can be verified to entail homomorphy identities.) Since the 2-element Boolean algebra as well as the real unit interval can be endowed with an MV-algebra structure, we obtain as natural corollaries two famous theorems: Arrow's theorem for judgement aggregation as well as McConway's [1981] characterisation of linear opinion pools.

Conceptually, this characterisation of aggregators can be seen as justifying a certain structuralist interpretation of social choice. Technically and perhaps more importantly, it opens up a new methodology to social choice theorists: the analysis of general aggregation problems by means of universal algebra.
\end{abstract}

Key words: propositional attitude aggregation; judgement aggregation; linear opinion pooling; Arrow's impossibility theorem; many-valued logic; MV-algebra; homomorphism; functional equation

2010 Mathematics Subject Classification: 91B14; 06D35.

Journal of Economic Literature classification: D71.

* It is a pleasure to thank Professor Christian List, Professor Bernard Monjardet, Professor Daniel Eckert as well as an anonymous referee for helpful comments on this paper and related earlier work. The preparation of the final version of this article was supported by a Visiting Fellowship of the Munich Center for Mathematical Philosophy funded by the Alexander von Humboldt Foundation.

$\dagger$ Institut für Mathematische Wirtschaftsforschung, Universität Bielefeld, Universitätsstraße 25, D-33615 Bielefeld, Germany. E-mail address: fherzberg@uni-bielefeld.de

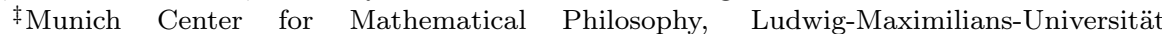
München, Geschwister-Scholl-Platz 1, D-80539 Munich, Germany. E-mail address: frederik.herzberg@lrz.uni-muenchen.de 


\section{Introduction}

Recently, Dietrich and List [2011] have proposed the fundamentals of a general theory of aggregation, with the aim of creating a unified theory of preference aggregation, judgement aggregation (cf. List and Puppe [2009] for a survey), probabilistic opinion pooling and more general many-valued ${ }^{1}$ aggregation problems. In a very general logical framework, Dietrich and List [2011] have proved that for sufficently complex aggregation problems, all independent and Paretian aggregators are already systematic. (Systematicity is a strong variant of independence of irrelevant alternatives; in the probabilistic opinion pooling literature following McConway [1981] it is known as the Strong Setwise Property.) In an earlier paper, Dietrich and List [2008] had already shown that judgement aggregation can be treated as a special case of generalised probabilistic opinion pooling and that in this setting, Arrow's dictatorial impossibility theorem for judgement aggregation is a special case of a generalisation of McConway's [1981] characterisation of linear opinion pools.

So far, however, no characterisations of general systematic many-valued propositional-attitude aggregators are known, nor has the case of infinite electorates been treated as yet. The present paper attempts to fill this gap: We generalise the main idea in Herzberg [2010] and prove that systematic manyvalued propositional-attitude aggregators are homomorphisms in the category of MV-algebras (for short: MV-homomorphisms) as defined by C.C. Chang [1958a]. Natural corollaries of this characterisation theorem (for the special case of a finite electorate) are both Arrow's dictatorial impossibility theorem for judgement aggregation and McConway's [1981] characterisation of linear opinion pools.

The proof idea for this characterisation theorem is the following: Any systematic aggregator can be reduced to a map which assigns truth values to truth-value profiles (i.e. sequences of truth values whose length is the cardinality of the population). In addition, this map will have to satisfy some functional equation. It turns out that this functional equation can be re-interpreted as the defining equation of some homomorphy concept, viz. homomorphy in the category of MV-algebras.

Informally speaking, our result says that systematic attitude aggregators are essentially the same as algebraically structure-preserving maps from truth-value profiles to truth values. In other words: The social (coalitional/power) structure entailed by systematic social-choice mechanisms has a structural resemblance to the set of truth values. Therefore, our result might have accomplished (using universal algebra - the epitome of a science of abstract structure) a rigorous justification for - what may be called - a structuralist view of social choice, viz. the intuition that "systematic aggregation rules link the power structure within the population with the logical structure underlying the agenda" (borrowing a phrase from Daniel Eckert ${ }^{2}$.

Structuralist interpretations of social choice aside, we hope that our result will be useful for yet another reason: It allows to reduce general aggregation problems to homomorphism classification problems; and since such

\footnotetext{
${ }^{1}$ We use the term 'many-valued' (as in 'many-valued logic') in the most inclusive sense i.e. encompassing the binary case - unless explicitly indicated otherwise. In other words, 'many-valued' is in general used as a synonym for 'pluri-valued' in this paper.

${ }^{2}$ Personal communication.
} 
problems have been studied extensively by algebraists, a vast literature of pure mathematical research might wait for discovery and straightforward application by social choice theorists.

It should be noted that the use of algebraic - in particular, lattice-theoretic and Boolean algebraic - methods has a long tradition in preference aggregation theory, cf. e.g. D.J. Brown [1974], the monographs by Kim and Roush [1980] or Aleskerov [1999] and the recent work of Daniëls and Pacuit [2009]. (A proof of Arrow's [1963] impossibility theorem using ultrafilters was published by Fishburn even as early as 1970.) Some authors have also employed filters and ultrafilters to establish impossibility theorems in judgement aggregation (e.g. Daniëls [2006], Dietrich and Mongin [2010] and Klamler and Eckert [2009]). Moreover, the relation between merging of opinions and certain functional equations - which, as was already remarked above, can often be interpreted as homomorphy relations - has long been recognised in the opinion pooling literature (cf. e.g. Aczél, Kannapan, Ng and Wagner [1983] and Aczél [1989]). Nevertheless, with the exception of the two aforementioned papers by Daniëls and Pacuit [2009] and Herzberg [2010], the published literature does not contain any systematic approaches to tackle general aggregation problems from a purely algebraic perspective. Our approach in this paper differs from Daniëls and Pacuit [2009] in that they start from a lattice-theoretic structure and then impose sufficient axioms to obtain an Arrow-style impossibility result, whereas we already assume an MV-algebra structure from the beginning and study the more general question of (algebraic) aggregator characterisation for electorates of arbitrary cardinality.

Hence, in this paper, we will first outline a formal framework for rather general many-valued aggregation problems by means of the notion of an MV-algebra (Section 2). We shall then list a number of assumptions, mainly generalisations of standard Arrovian responsiveness axioms for the aggregation functions (Section 3). Thereafter, we shall state a characterisation theorem for aggregators as MV-algebra homomorphisms and derive two wellknown corollaries from judgement aggregation and probabilistic opinion pooling (Section 4); the proofs can be found in an appendix. Possible extensions of our methodology are discussed in the final Section 5

\section{Formal framework}

In the following, we describe a formal model for the aggregation of many-valued propositional attitudes. The electorate will be given by some (finite or infinite) set $N$. In addition, a set of propositions $X$ (agenda) in a sufficiently expressive language will be fixed, and the electorate as well as each individual will be supposed to display a certain attitude towards each proposition in the agenda (thus assigning a truth value). The set of possible attitudes or truth values will be denoted $M$ (and will be assumed to possess some additional structure, viz. that of an MV-algebra). Thus, each individual expresses his or her attitudes towards the elements of the agenda through a function from $X$ to $M$, called attitude function. Then the attitudes of all individuals can be captured by an $N$-sequence of attitude functions (i.e. by a map from $N$ to $M^{X}$ ); $\operatorname{such}$ an $N$ sequence will be called profile. An aggregator is then simply a map from (a suitable subset of) the set of profiles to the set of attitude functions. 


\subsection{Agenda syntax}

Let $\mathcal{L}$ be the language of many-valued propositional logic. In other words, let $\mathcal{L}$ be the language whose symbols consist of countably many propositional variables, a propositional constant 0 (falsehood), a binary operation $\oplus$ (strong disjunction) and a unary operator $\neg$ (negation). The set of well-formed formulae in this language shall be denoted $\mathbf{L}$.

The idea behind the introduction of a strong disjunction is that in manyvalued logic has to do with the possibility of partial assent to any given proposition in many-valued logic. Now, if someone who only gives partial assent to both $p$ and $q$ is asked about her assessment of ' $p$ or $q$ ', she has two options: She may either, roughly speaking, assume that $p$ and $q$ hold true in disjoint sets of worlds and thus additively combine the partial assent — or she may adopt a more cautious stance and only assign as much assent as she would have given to either of $p$ and $q$. The first interpretation connects $p$ and $q$ by a strong disjunction, the latter by a weak disjunction.

A number of standard abbreviations will be helpful. First, we define a new propositional constant 1 (truth) by $\neg 0$. Next, we define additional operations. The operation of weak disjunction (denoted $\vee$ ) will be defined via

$$
\forall p, q \in \mathbf{L} \quad p \vee q=\neg(\neg p \oplus q) \oplus q,
$$

and strong conjunction (denoted $\otimes$ ) as well as weak conjunction $(\operatorname{denoted} \wedge$ ) can then be defined through De Morgan's laws:

$$
\begin{aligned}
\forall p, q \in \mathbf{L} \quad p \otimes q & =\neg(\neg p \oplus \neg q), \\
p \wedge q & =\neg(\neg p \vee \neg q) .
\end{aligned}
$$

The implication operation $($ denoted $\rightarrow$ ) can be defined as

$$
p \rightarrow q=\neg p \oplus q
$$

(In their original paper, Łukasiewicz and Tarski [1930] took $\neg$ and $\rightarrow$ as the primitive logical symbols of their language.) Eukasiewicz logic is then given by the provability relation $\vdash$, given by modus ponens (i.e. for all $p, q \in \mathbf{L}$ and $S \subseteq \mathbf{L}$, if $S \vdash p$ and $S \vdash(p \rightarrow q)$, then $S \vdash q)$ and the following axiom schemes: For all $p, q \in \mathbf{L}$,

A1. For all $p, q \in \mathbf{L}$, the proposition $p \rightarrow(q \rightarrow p)$ is an axiom.

A2. For all $p, q \in \mathbf{L}$, the proposition $(p \rightarrow q) \rightarrow((q \rightarrow r) \rightarrow(p \rightarrow r))$ is an axiom.

A3. For all $p, q \in \mathbf{L}$, the proposition $((p \rightarrow q) \rightarrow q) \rightarrow((q \rightarrow p) \rightarrow p)$ is an axiom.

A4. For all $p, q \in \mathbf{L}$, the proposition $(\neg p \rightarrow \neg q) \rightarrow(q \rightarrow p)$ is an axiom.

(Cf. Rose and Rosser [1958] and Chang [1958b].)

One can define a relation $\equiv$, called provable equivalence, on $\mathbf{L}$ by saying that $p$ is provably equivalent to $q$ (denoted $p \equiv q)$ if and only if both $\vdash(p \rightarrow q)$ as well as $\vdash(q \rightarrow p)$ (wherein $\vdash p$ is, for all $p \in \mathbf{L}$, shorthand for $\varnothing \vdash p$ ). It is not difficult to verify that $\equiv$ is an equivalence relation on $\mathbf{L}$. The set of equivalence 
classes shall be denoted $\mathbf{L} / \equiv$. Representative-wise, one can define the constant 0 , the operator $\neg$ and the operation $\oplus$ on $\mathbf{L} / \equiv$; again, it is not hard to prove that these are well-defined. Therefore, the operations $\otimes, \vee, \wedge, \rightarrow$ can be defined on $\mathbf{L}$ as well.

\section{$2.2 \quad$ Agenda semantics}

Recall that an MV-algebra $M$ is a structure $(M, \oplus, \neg, 0)$ such that $(M, \oplus, 0)$ is a commutative monoid (i.e. $\oplus$ is a commutative and associative binary operation on $M$ with neutral element 0 ) and the following identities are satisfied for all $x, y \in M$ :

- $\neg \neg x=x$,

- $x \oplus 1=1$,

- $x \vee y=y \vee x$,

wherein 1 is shorthand for $\neg 0$ and $\vee$ is defined via

$$
\forall x, y \in M \quad x \vee y=\neg(\neg x \oplus y) \oplus y .
$$

It turns out that the structure $(\mathbf{L} / \equiv, \oplus, \neg, 0)$, i.e. the set of equivalence classes of provably equivalent formulae from many-valued propositional logic with the canonical operations, is an MV-algebra, the so-called Lindenbaum algebra for Łukasiewicz's many-valued logic. This observation allows us to take an algebraic approach to the semantics of many-valued propositional logic, essentially due to C.C. Chang [1958a, 1959]: Let us henceforth assume that the truth values form an MV-algebra; we shall hence fix an MV-algebra $M$ for the rest of this paper and shall refer to it as the set of truth values. Under these hypotheses, an $M$-valuation can be defined as an MV-algebra homomorphism from $\mathbf{L} / \equiv$ to $M$. If $I$ is an $M$-valuation and $p \in \mathbf{L}$, we shall usually simply write $I(p)$ instead of $I\left([p]_{\equiv}\right)$.

Important examples of MV-algebras are the following (cf. already Chang [1958a]):

- Any Boolean algebra is an MV-algebra (with the lattice operations $\vee$ and $\wedge$ playing the rôles of $\oplus$ and $\otimes$ ).

- If $M=[0,1]$, the set of all real numbers between 0 and 1 , one obtains an MV-algebra with zero element 0 by setting $\neg x=1-x$ and $x \oplus y=$ $\min \{x+y, 1\}$ for all $x, y \in[0,1]$. This is called the standard $M V$-algebra. It is the set of truth values for the infinite-valued logic $L_{\beth_{1}}$. The derived operations are given by

$$
x \otimes y=\max \{0, x+y-1\}, \quad x \vee y=\max \{x, y\}, \quad x \wedge y=\min \{x, y\} .
$$

- With the same definitions for $0, \neg$ and $\oplus$, the set $M=[0,1] \cap \mathbf{Q}$ (the set of all rational numbers between 0 and 1) is an MV-algebra; it is the set of truth values for Łukasiewicz's infinite-valued logic $L_{\aleph_{0}}$.

- Again with the same definitions for $0, \neg$ and $\oplus$, the set $M=$ $\{0,1 / m, \ldots,(m-1) / m, 1\}$ is an MV-algebra for every positive integer $m$. It is the set of truth values for Eukasiewicz's $(m+1)$-valued logic $L_{m+1}$. 
Rose and Rosser [1958] and Chang [1959] have shown, each by a different method, the completeness of $L_{\aleph_{0}}$.

\subsection{Attitude functions}

Consider a set $X \subseteq \mathbf{L}$, henceforth called the agenda. Attitude functions are functions from $X$ to $M$. An attitude function $A$ is rational if and only if it can be extended to an $M$-valuation, i.e. there exists an $M$-valuation $I$ such that $A(p)=I\left([p]_{\equiv}\right)$ for all $p \in X$. Therefore, any rational attitude function $A$ is also well-defined not only on $X$, but on the closure of $X$ under $\neg$ and $\oplus$. A (rational) profile is an $N$-sequence of (rational) attitude functions; our typical notion for a profile will be $\underline{A}=\left(A_{i}\right)_{i \in N}$. An attitude aggregator is a map from a subset of the set of profiles to the set of attitude functions. An attitude aggregator $F$ is a dictatorship if and only if there exists some $i \in N$ such that $F(\underline{A})=A_{i}$ for all $\underline{A}$ in the domain of $F$.

An important observation is the extendibility of rational attitude functions:

Remark 1. Denote the closure of the agenda $X$ under $\neg$ and $\oplus$ by $Y$. Any rational attitude function can be uniquely extended to a function from $Y$ to $M$.

We shall always identify the extension of any rational attitude function $A$ with its extension to $Y$. Thus, in the following, all rational attitude functions are assumed to be defined on the whole of $Y$. The reason for not choosing $X$ to be closed under $\neg$ and $\oplus$ from the beginning is conceptual rather than technical: It should be possible to interpret consistent subsets of the agenda as sets of "premisses" (cf. e.g. Dietrich and Mongin [2010]). While such sets of premisses will typically already determine the truth values of many more (complex) propositions, only the premisses will be subject to a collective decision mechanism.

\subsection{Examples}

The simplest example for this framework is the "classical" propositional judgement aggregation, where $M$ is $2=\{0,1\}$ endowed with the Boolean algebra structure. Another example would be probabilistic opinion pooling: Here, $M$ is the standard MV-algebra $[0,1]$, and the agenda consists of propositional constants for each atomic event and each complement of an atomic event. In addition, this framework encompasses aggregation problems with respect to more general many-valued logics: All that is required is that the set of truth values forms an MV-algebra; by that means, the framework proposed in this paper covers aggregation of propositions in a large class of finite- and infinitevalued logics as well. For example, voting with abstentions can easily be modeled as an aggregation problem in a three-valued logic, e.g. Łukasiewicz's $L_{3}$.

\section{Aggregator responsiveness axioms}

In this section, we generalise (mostly standard) terminology from aggregation theory, in order to be able to formulate our subsequent results on propositionalattitude aggregators. We shall use the abbrevation

$$
\underline{A}(p)=\left(A_{i}(p)\right)_{i \in N}
$$


for all propositions $p \in X$ and all profiles $\underline{A} \in\left(M^{X}\right)^{N}$.

Definition 2. An attitude aggregator $F$ is rational if and only if for all rational profiles $\underline{A}$ in the domain of $F, F(\underline{A})$ is a rational attitude function.

Definition 3. An attitude aggregator $F$ is universal if and only if its domain comprises all rational profiles.

Independent aggregation means that the aggregate attitude towards any proposition $p$ does not depend on the individuals' attitudes towards propositions other than $p$ :

Definition 4. An attitude aggregator $F$ is independent if and only if there exists a map $G: M^{N} \times X \rightarrow M$ such that for all profiles $\underline{A}$ in the domain of $F$ and for all $p \in X, F(\underline{A})(p)=G(\underline{A}(p), p)$.

Systematic aggregation is a special case of independent aggregation, where $G$ is constant in the second argument, i.e. the aggregate attitude towards any proposition $p$ only depends on $p$ through the individuals' attitudes towards $p$ :

Definition 5. An attitude aggregator $F$ is systematic if and only if there exists a map $f: M^{N} \rightarrow M$, called decision criterion of $F$, such that for all profiles $\underline{A}$ in the domain of $F$ and for all $p \in X$,

$$
F(\underline{A})(p)=f(\underline{A}(p)) .
$$

Under a mild additional assumption, the decision criterion of a systematic aggregator is unique. Let us call a formula $p \in \mathbf{L}$ strictly contingent if and only if there exists for all $x \in M$ some $M$-valuation $I$ with $I(p)=x$.

Remark 6. If the agenda contains some strictly contingent sentence $p_{0}$, then any universal systematic attitude aggregator $F$ has a unique decision criterion.

Though it appears much stronger at first sight, systematicity is under mild conditions actually equivalent to independence (cf. Dietrich and List [2011, Theorem 2]). At least as strong is the following notion (recall that rational attitude functions can be uniquely extended to the closure of the agenda under $\neg$ and $\oplus$ ):

Definition 7. A systematic attitude aggregator $F$ is strongly systematic if and only if Equation (1) holds even for all $p$ in the closure of $X$ under $\neg$ and $\oplus$ and all profiles $\underline{A}$ in the domain of $F$.

If the agenda is closed under $\neg$ and $\oplus$, then systematicity and strong systematicity trivially coincide.

The Pareto principle asserts that any proposition which is rejected unanimously by all individuals, must be collectively rejected:

Definition 8. An attitude aggregator $F$ is Paretian if and only if for all profiles $\underline{A}$ in the domain of $F$ and all $p \in X$, if $A_{i}(p)=0$ for all $i \in N$ (i.e. $\underline{A}(p)=\underline{0}$ ), then $F(\underline{A})(p)=0$.

For most of the paper, we need to impose additional assumptions on the logical expressivity or complexity of the agenda. 
Definition 9. The agenda $X$ is called complex if and only if there exists a strictly contingent proposition $p_{0}$ in $X$ and there also exist strictly contingent propositions $p_{1}, p_{2}, p_{3}, q_{1}, q_{2}$ in the closure of $X$ under $\neg$ and $\oplus$ such that for all $M$-valuations $I$, one has $I\left(p_{1}\right) \oplus I\left(p_{2}\right)=I\left(p_{3}\right)$ and $\neg I\left(q_{1}\right)=I\left(q_{2}\right)$. If $p_{1}, p_{2}, p_{3}, q_{1}, q_{2}$ are even in $X$, then $X$ is said to be rich.

The point about rich (complex) is that one can generate manifold combinations of truth values through $M$-valuations of elements of the agenda (the closure of the agenda under $\neg$ and $\oplus$, respectively) only. (For example, $\{p, \neg p, q, \neg q, r, \neg r, s, \neg s, t, \neg t\}$ is always a rich agenda if $p, q, r, s, t$ are propositional constants, but $\{p, \neg p\}$ is not.) This aspect of agenda complexity or richness will be the key to the proof of our main result, via the notion of strongly systematisable aggregators:

Definition 10. In this paper, a systematic attitude aggregator $F$ for a complex agenda $X$ is called strongly systematisable if and only if $F$ is either strongly systematic or the agenda $X$ is rich.

\section{Results}

Note that $M^{N}$ is - as the direct product of $\operatorname{card}(N)$ identical copies of $M-$ again an MV-algebra; the strong disjunction $\oplus_{N}$ and negation $\neg_{N}$ are defined componentwise, the zero element $0_{N}$ is just the $N$-sequence $\underline{0}$ of 0 's.

Our main theorem establishes a one-to-one correspondence between maps from $M^{N}$ to $M$ that preserve the algebraic structure and such attitude aggregators as are rational, universal, Paretian and strongly systematisable. Its morale is the following: Very roughly speaking, every decision criterion that is algebraically well-behaved gives rise to a "nice" aggregator; and conversely, any "nice" aggregator can be reduced to a decision criterion that is algebraically well-behaved.

Theorem 11. If $F$ is a rational, universal, Paretian and strongly systematisable attitude aggregator, then the decision criterion of $F$ is an $M V$-homomorphism.

Conversely, if $f$ is an $M V$-homomorphism and $F$ is defined by Equation (1) for all rational profiles $\underline{A}$ and all $p \in X$, then $F$ is a rational, universal, Paretian and systematic attitude aggregator.

(The uniqueness of the decision criterion had already been noted in Remark 6.

If $M=\{0,1\}$ with the usual Boolean structure, then $M^{N}$ is again a Boolean algebra and isomorphic to the power-set Boolean algebra of $N$. This allows us to deduce, as an easy corollary to Theorem 11, the recent result in Herzberg [2010]. Ultimately, this leads to Arrow's impossibility theorem for judgement aggregation (recall the previous remark about the equivalence of systematicity and independence under mild conditions), first proved by List and Pettit [2002].

Corollary 12. Suppose $F$ is a rational, universal, Paretian and strongly systematisable attitude aggregator. If the algebra of truth values is Boolean, then the decision criterion of $F$ is a Boolean homomorphism.

If the algebra of truth values is just the Boolean algebra $\{0,1\}$ and the electorate $N$ is finite, then $F$ is a dictatorship. 
If $M$ is the standard MV-algebra, then Theorem 11 yields McConway's [1981] characterisation of linear opinion pools ("weighted averaging") as a second corollary:

Corollary 13. Let $F$ be a rational, universal, Paretian and strongly systematisable aggregator, let the algebra of truth values be the standard $M V$ algebra [0,1], and suppose the electorate $N$ is finite. Then the decision criterion of $F$ is a linear map from $[0,1]^{N}$ to $[0,1]$.

In less abstract terms: In the setting of Corollary 13 (well-behaved aggregation of information encoded in fuzzy logic with a finite electorate), profiles will be sequences of maps from the agenda to the unit interval $[0,1]$, and there will be a vector of weights $\left(\alpha_{i}\right)_{i \in N} \in[0,1]^{N}$ such that $\sum_{i \in N} \alpha_{i}=1$ and

$$
F(\underline{A})(p)=\sum_{i \in N} \alpha_{i} A_{i}(p)
$$

for all $\underline{A}$ and all $p$ in the agenda.

\section{Discussion}

We have seen that one can neatly formulate an aggregation theory for general many-valued propositional attitudes based on the theory of MV-algebras. Aggregators satisfying common responsiveness axioms (agenda complexity resp. richness, collective rationality, universality, systematicity resp. strong systematicity, Pareto principle) then simply correspond to MV-homomorphisms - algebraically structure-preserving maps — from $M^{N}$ to $M$ ( $M$ being the MV-algebra of truth values). For special cases of $M$, one can use classical classification results for such homomorphisms to obtain a classification of Paretian systematic aggregators, e.g. if $M$ is the Boolean algebra $2=$ $\{0,1\}$ (which leads to the judgement-aggregation analogue of Arrow's [1963] impossibility theorem, cf. Dietrich and List [2007]) or if $M=[0,1]$ (which entails McConway's [1981] characterisation of linear opinion pooling). More general aggregator classifications might be derived from MV-algebra classifications (cf. Chang [1959], Mundici [1986], Cignoli and Mundici [1997]).

Informally speaking, we have shown that systematic attitude aggregators will always preserve a certain algebraic structure that is found both on the set of profiles (of attitude functions) and the set of attitude functions itself: the MV-algebra structure. Since such structure-preserving maps can be classified in many instances, impossibility theorems and possibility theorems can be obtained as corollaries via results from pure universal algebra. In particular, since systematicity and independence of irrelevant alternatives are equivalent under mild conditions in general aggregation contexts (as shown by Dietrich and List [2011]) and since $M=\{0,1 / m, \ldots,(m-1) / m, 1\}$ is an MV-algebra for every positive integer $m$ ), our result can probably be utilised to give a shorter, arguably more direct proof of Pauly and van Hees' [2006] impossibility theorems for propositional-attitude aggregation in finite-valued logic with finite electorates. It would be enough to know that projections are the only MVhomomorphisms from the Cartesian power $M^{N}$ to $M$ in that setting (provided $N$ is finite). For propositional-attitude aggregation in infinite-valued logics, there are possibility theorems even for finite electorates: For instance, if 
the underlying logic is the infinite-valued $\operatorname{logic} L_{\beth_{1}}$ (i.e. the truth values may be drawn arbitrarily from the standard MV-algebra $M=[0,1]$ ), the resulting aggregation problems is essentially a reformulation of probabilistic opinion pooling, and in this setting there exist - even for finite electorates - continuum-many structure-preserving maps from $M^{N}$ to $M$ and thus uncountably many non-dictatorial systematic aggregators (viz. all weightedaverage rules). It would be interesting to see if impossibility theorems can also be avoided for finite electorates in tukasiewicz's infinite-valued logic $L_{\aleph_{0}}$. In light of the results in this paper, this boils down to a purely algebraic question: Are there MV-homomorphisms other than projections from the Cartesian power $M^{N}$ to $M$ if $M=[0,1] \cap \mathbf{Q}$ and $N$ is finite?

Our algebraic approach to aggregation theory could be taken further by allowing for Heyting algebras or BL-algebras as sets of truth values. By that means, aggregation of intuitionistic resp. fuzzy propositional attitudes could be studied in full generality.

A powerful alternative to algebraic aggregation theory is the model-theoretic approach pioneered by Lauwers and Van Liedekerke [1995], as it allows to study aggregation problems for predicate logic in a natural manner as well. (Cf. also Herzberg, Lauwers, Van Liedekerke and Fianu [2010] for an addendum as well as Herzberg and Eckert [2011a, 2011b] for a generalisation.) It remains to be seen whether even many-valued aggregation problems can be studied by model-theoretic methods; such an approach could pave the way for a systematic analysis of aggregation problems in many-valued predicate logic. The algebraic approach to many-valued model theory proposed by Zlatoš [1981] might be a first starting point for such an endeavour. 


\section{Appendix}

\section{A Proofs}

Proof of Remark 1. Let $A$ be a rational attitude function. Consider any two $M$-valuations $I, I^{\prime}$ such that $A(p)=I(p)=I^{\prime}(p)$ for all $p \in X$ or, more precisely, $A(p)=I\left([p]_{\equiv}\right)=I^{\prime}\left([p]_{\equiv}\right)$ for all $p \in X$. In other words, $I$ and $I^{\prime}$ are homomorphisms from $\mathbf{L} / \equiv$ to $M$ which agree on the set $[X]_{\equiv}$ of $\equiv$ equivalence classes of elements of $X$. Thus, they must agree on the closure of $[X]_{\equiv}$ under the operations $\neg$ and $\oplus$ in $\mathbf{L} / \equiv$. Call this closure $[Y]_{\equiv}$. Since the operations $\neg$ and $\oplus$ in $\mathbf{L} / \equiv$ are defined representative-wise, $[Y]_{\equiv}$ equals the set of equivalence classes of elements of $Y$ (the closure of $X$ under the operations $\neg$ and $\oplus$ in $\mathbf{L})$. It follows that $I\left([p]_{\equiv}\right)=I^{\prime}\left([p]_{\equiv}\right)$ for all $p \in Y$.

Proof of Remark 6. Let $p_{0}$ be as in the statement of Remark 6, let $f$ and $f^{\prime}$ be decision criteria of $F$, and let $\underline{x}=\left(x_{i}\right)_{i \in N} \in M^{N}$. Then there exists for each $i \in N$ some $M$-valuation $I_{i}$ such that $I_{i}\left(p_{0}\right)=x_{i}$. Now each $I_{i}$ induces a rational attitude function $A_{i}$ defined by $A_{i}(p)=I_{i}(p)$ for all $p \in X$, so that in particular $A_{i}\left(p_{0}\right)=x_{i}$ for all $i \in M$. As $F$ is universal, the profile $\underline{A}=\left(A_{i}\right)_{i \in N}$ is in the domain of $F$. Hence

$$
f(\underline{x})=f\left(\underline{A}\left(p_{0}\right)\right)=F(\underline{A})\left(p_{0}\right)=f^{\prime}\left(\underline{A}\left(p_{0}\right)\right)=f^{\prime}(\underline{x}) .
$$

Proof of Theorem 11. Let $F$ be a rational, universal, Paretian and strongly systematisable attitude aggregator, and let $f$ be the decision criterion of $F$.

Consider any two elements of $M^{N}, \underline{x}=\left(x_{i}\right)_{i \in N}$ and $y=\left(y_{i}\right)_{i \in N}$. Let $p_{1}, p_{2}, p_{3}, q_{1}, q_{2}$ be as in the definition of agenda complexity. Then on the one hand, since $p_{1}, p_{2}, p_{3}, q_{1}, q_{2}$ are strictly contingent by assumption, there exists for each $i \in N$ some $M$-valuations $I_{i}, I_{i}^{\prime}, I_{i}^{\prime \prime}$ such that

- $I_{i}\left(p_{1}\right)=x_{i}$ and $I_{i}\left(p_{2}\right)=y_{i}$,

- $I_{i}^{\prime}\left(q_{1}\right)=x_{i}$,

- $I_{i}^{\prime \prime}\left(p_{1}\right)=0$.

On the other hand, since $p_{1}, p_{2}, p_{3}, q_{1}, q_{2}$ were assumed to be as in the definition of agenda complexity, it follows for each $i \in N$,

- not only $I_{i}\left(p_{1}\right)=x_{i}$ and $I_{i}\left(p_{2}\right)=y_{i}$, but also $I_{i}\left(p_{3}\right)=x_{i} \oplus y_{i}$ and $I\left(p_{3}\right)=I\left(p_{1}\right) \oplus I\left(p_{2}\right)$ for every $M$-valuation $I$,

- not only $I_{i}^{\prime}\left(q_{1}\right)=x_{i}$, but also $I_{i}^{\prime}\left(q_{2}\right)=\neg x_{i}$ and $I^{\prime}\left(q_{2}\right)=\neg I^{\prime}\left(q_{1}\right)$ for every $M$-valuation $I^{\prime}$,

- $I_{i}^{\prime \prime}\left(p_{1}\right)=0$.

In other words, there exists an $N$-sequence $\underline{I}=\left(I_{i}\right)_{i \in N}$ of $M$-valuations such that

- $\underline{I}\left(p_{1}\right)=\underline{x}, \underline{I}\left(p_{2}\right)=\underline{y}, \underline{I}\left(p_{3}\right)=\underline{x} \oplus_{N} y$ and $I\left(p_{3}\right)=I\left(p_{1}\right) \oplus I\left(p_{2}\right)$ for every $M$-valuation $I$, 
- $\underline{I}^{\prime}\left(q_{1}\right)=\underline{x}, \underline{I}^{\prime}\left(q_{2}\right)=\neg N \underline{x}$ and $I^{\prime}\left(q_{2}\right)=\neg I^{\prime}\left(q_{1}\right)$ for every $M$-valuation $I^{\prime}$,

- $\underline{I}^{\prime \prime}\left(p_{1}\right)=\underline{0}=0_{N}$.

Next note that by restricting each $I_{i}, I_{i}^{\prime}$ and $I_{i}^{\prime \prime}$ to the set of equivalence classes of elements of $X$ (recall that $I(p)$ is shorthand for $I([p] \equiv)$ for any $M$-valuation $I$ and any $p \in \mathbf{L}$ ), one obtains rational attitude functions $A_{i}, A_{i}^{\prime}$ and $A_{i}^{\prime \prime}$. All $A_{i}$, $A_{i}^{\prime}$ and $A_{i}^{\prime \prime}$ are rational attitude functions and thus can be uniquely extended to $Y$ by Remark 11. Hence, we have constructed rational profiles $\underline{A}=\left(A_{i}\right)_{i \in N}$, $\underline{A}^{\prime}=\left(A_{i}^{\prime}\right)_{i \in N}$ and $\underline{A}^{\prime \prime}=\left(A_{i}^{\prime \prime}\right)_{i \in N}$ such that

- $\underline{A}\left(p_{1}\right)=\underline{x}, \underline{A}\left(p_{2}\right)=\underline{y}, \underline{A}\left(p_{3}\right)=\underline{x} \oplus_{N} \underline{y}$, and $I\left(p_{3}\right)=I\left(p_{1}\right) \oplus I\left(p_{2}\right)$ for every $M$-valuation $I$,

- $\underline{A}^{\prime}\left(q_{1}\right)=\underline{x}, \underline{A^{\prime}}\left(q_{2}\right)=\neg N \underline{x}$, and $I^{\prime}\left(q_{2}\right)=\neg I^{\prime}\left(q_{1}\right)$ for every $M$-valuation $I^{\prime}$,

- $\underline{A}^{\prime \prime}\left(p_{1}\right)=0_{N}$.

Note that since $F$ is universal, the profiles $\underline{A}, A^{\prime}, \underline{A}^{\prime \prime}$ must be in the domain of $F$. Since $F$ is rational, $F(\underline{A}), F\left(\underline{A}^{\prime}\right)$ and $F\left(\underline{A}^{\prime \prime}\right)$ are rational attitude functions and thus can be uniquely extended to $Y$ by Remark 1. Moreover, there exist $M$-valuations $I$ and $I^{\prime}$ such that $F(\underline{A})(p)=I(p)$ as well as $F\left(\underline{A}^{\prime}\right)(p)=I^{\prime}(p)$ for all $p \in X$ and hence, by the homomorphy of $I$, also for all $p \in Y$. From here, it follows that

- $F(\underline{A})\left(p_{3}\right)=F(\underline{A})\left(p_{1}\right) \oplus F(\underline{A})\left(p_{2}\right)$,

- $F\left(\underline{A}^{\prime}\right)\left(q_{2}\right)=\neg F\left(\underline{A}^{\prime}\right)\left(q_{1}\right)$.

Let us next exploit the choice of $p_{1}, p_{2}, p_{3}, q_{1}, q_{2}$ as in the definition of agenda complexity and the strong systematicity of $F$ or the richness of $X$. This yields for any $M$-valuation $I$ which extends $F(\underline{A})$,

$$
\begin{aligned}
f\left(\underline{x} \oplus_{N} \underline{y}\right) & =f\left(\underline{A}\left(p_{3}\right)\right)=I\left(p_{3}\right) \\
& =I\left(p_{1}\right) \oplus I\left(p_{2}\right)=f\left(\underline{A}\left(p_{1}\right)\right) \oplus f\left(\underline{A}\left(p_{2}\right)\right)=f(\underline{x}) \oplus f(\underline{y}) .
\end{aligned}
$$

Similarly (this time applying the formulae in the definition of agenda complexity to an $M$-valuation $I$ which extends $\left.F\left(\underline{A}^{\prime}\right)\right)$,

$$
\begin{aligned}
f(\neg N \underline{x}) & =f\left(\underline{A}^{\prime}\left(q_{2}\right)\right)=I\left(q_{2}\right) \\
& =\neg I\left(q_{1}\right)=\neg f\left(\underline{A}^{\prime}\left(q_{1}\right)\right)=\neg f(\underline{x}) .
\end{aligned}
$$

Thus, $f$ preserves the operators $\neg$ and $\oplus$ and maps the zero element $0_{N}$ of $M^{N}$ to $0 \in M$; hence, $f$ is an MV-homomorphism.

Conversely, let $f$ be an MV-homomorphism. Clearly, the $F$ defined by Equation (1) for all rational profiles $\underline{A}$ and all $p \in X$ is both systematic and universal. Moreover, since $f$ is a homomorphism, any composition of $f$ with an $N$-sequence of MV homomorphisms from $\mathbf{L}$ to $M$ will again be a homomorphism from $\mathbf{L}$ to $M$. In other words, the composition of $f$ with an $N$-sequence of valuations is again a valuation. This shows that the composition of $f$ with a rational profile is a rational attitude function. Hence, the $F$ defined by Equation (1) is rational. Since $f\left(0_{N}\right)=f(\underline{0})=0$, it is clear that $F$ is Paretian. 
Proof of Corollary 12. If $M$ is even a Boolean algebra, then so is $M^{N}$. By Theorem 11, the decision criterion $f$ is an MV-homomorphism. Since any MV-homomorphism between two Boolean algebras is a Boolean homomorphism (because the Boolean operations $\vee$ and $\wedge$ as well as the constant 1 can be defined through $\neg$ and $\oplus: x \vee y=\neg(\neg x \oplus y) \oplus y$ and $x \wedge y=\neg(\neg x \vee \neg y)$ for all $x, y$ and $1=\neg 0)$, it follows that $f$ is actually a Boolean homomorphism. Boolean algebra teaches that the shell of $f$, i.e. $f^{-1}\{1\}=f^{-1}\{\neg 0\}$, is a filter in $2^{N}$ (which is isomorphic to the power-set Boolean algebra of $N$ ), and if $M=\{0,1\}$, then the shell of $f$ is even an ultrafilter on $N$. Now if $N$ is finite, this means - as all ultrafilters on finite sets are principal - that there exists some $i_{0} \in N$ such that $f^{-1}\{1\}=\left\{C \subseteq N: i_{0} \in C\right\}$. This, however, implies that $F$ is a dictatorship, the dictator being $i_{0}$.

Proof of Corollary 13. Without loss of generality, we may assume $N=$ $\{1, \ldots, n\}$ for some positive integer $n$. By Theorem 11, the decision criterion $f:[0,1]^{N} \rightarrow[0,1]$ is an MV-homomorphism. This implies, if $M$ is the standard MV-algebra $[0,1]$, that

$$
f\left(x_{1}, \ldots, x_{n}\right) \oplus f\left(y_{1}, \ldots, y_{n}\right)=f\left(x_{1} \oplus y_{1}, \ldots, x_{n} \oplus y_{n}\right)
$$

for all $x_{1}, y_{1}, \ldots, x_{n}, y_{n} \in[0,1]$. Hence (by the definition of $\oplus$ in the Eukasiewicz algebra, i.e. $x \oplus y=\min \{x+y, 1\}$ for all $x, y \in[0,1]$ and the componentwise definition of $\oplus$ in the direct power $\left.[0,1]^{N}\right)$ one has for all $x_{1}, y_{1}, \ldots, x_{n}, y_{n} \in[0,1]$ with $x_{i}+y_{i} \leq 1$ for all $i \in N$,

$$
f\left(x_{1}, \ldots, x_{n}\right)+f\left(y_{1}, \ldots, y_{n}\right)=f\left(x_{1}+y_{1}, \ldots, x_{n}+y_{n}\right) .
$$

One can now emulate McConway's [1981] original argument: An iterated application of the preceding equation yields for all $z_{1}, \ldots, z_{n} \in[0,1]$,

$$
\begin{aligned}
f\left(z_{1}, \ldots, z_{n}\right) & =f\left(z_{1}, 0, \ldots, 0\right)+f\left(0, z_{2}, \ldots, z_{n}\right) \\
& =f\left(z_{1}, 0, \ldots, 0\right)+f\left(0, z_{2}, 0, \ldots, 0\right)+f\left(0,0, z_{3}, \ldots, z_{n}\right) \\
& =\sum_{i=1}^{n} f(\underbrace{0, \ldots, 0}_{i-1}, z_{i}, \underbrace{0, \ldots, 0}_{n-i}) .
\end{aligned}
$$

Hence, definining $f_{i}$ by

$$
f_{i}(z)=f(\underbrace{0, \ldots, 0}_{i-1}, z, \underbrace{0, \ldots, 0}_{n-i})
$$

for every $z \in[0,1]$ and each $i \in N$, we obtain

$$
f\left(z_{1}, \ldots, z_{n}\right)=\sum_{i=1}^{n} f_{i}\left(z_{i}\right)
$$

for all $z_{1}, \ldots, z_{n} \in[0,1]$. Moreover, Equation (2) also implies $f_{i}(x+y)=$ $f_{i}(x)+f_{i}(y)$ for all $x, y \in[0,1]$ with $x+y \leq 1$ and each $i \in N$. Therefore, every $f_{i}$ satisfies Cauchy's functional equation. Also, the range of every $f_{i}$ is by definition contained in the range of $f$ and thus in $[0,1]$, whence $f_{i}(x)$ is 
nonnegative for all $x \in[0,1]$ and every $i \in N$. Therefore, there exists for every $i \in N$ some $\alpha_{i}$ such that $f_{i}(x)=\alpha_{i} x$ for all $x \in[0,1]$ (cf. Aczél [1961, 1966, Section 2.1.1, Theorem 1]), and this $\alpha_{i}$ must be nonnegative. Thus, $f\left(z_{1}, \ldots, z_{n}\right)=\sum_{i=1}^{n} \alpha_{i} z_{i}$ for all $z_{1}, \ldots, z_{n} \in[0,1]$.

\section{References}

Aczél, J. (1961). Vorlesungen über Funktionalgleichungen und ihre Anwendungen, volume 25 of Lehrbücher und Monographien aus dem Gebiete der exakten Wissenschaften. Mathematische Reihe. Birkhäuser, Basel.

Aczél, J. (1966). Lectures on functional equations and their applications, volume 19 of Mathematics in Science and Engineering. Academic Press, New York.

Aczél, J. (1989). Basics of functional equations arising from recent applications to economics and to other sciences. In XII Symposium on Operations Research (Passau, 1987), volume 59 of Methods of Operations Research, pages 3-14. Athenäum/Hain/Hanstein, Königstein.

Aczél, J., Kannappan, P., Ng, C., and Wagner, C. (1983). Functional equations and inequalities in "rational group decision making". In General inequalities, 3 (Oberwolfach, 1981), volume 64 of Internationale Schriftenreihe zur Numerischen Mathematik, pages 239-245. Birkhäuser, Basel.

Aleskerov, F. (1999). Arrovian aggregation models, volume 39 of Theory and Decision Library. Series B: Mathematical and Statistical Methods. Kluwer Academic Publishers, Boston, MA.

Arrow, K. (1963). Social choice and individual values, volume 12 of Cowles Commission Monographs. Wiley, New York, 2 edition.

Brown, D. (1974). An approximate solution to Arrow's problem. Journal of Economic Theory, 9(4):375-383.

Chang, C. (1958a). Algebraic analysis of many valued logics. Transactions of the American Mathematical Society, 88:467-490.

Chang, C. (1958b). Proof of an axiom of tukasiewicz. Transactions of the American Mathematical Society, 87:55-56.

Chang, C. (1959). A new proof of the completeness of the Łukasiewicz axioms. Transactions of the American Mathematical Society, 93:74-80.

Cignoli, R. and Mundici, D. (1997). An invitation to Chang's MV algebras. In Droste, M. and Göbel, R., editors, Advances in algebra and model theory (Essen, 1994; Dresden, 1995), volume 9 of Algebra Logic Appl., pages 171197. Gordon and Breach, Amsterdam.

Daniëls, T. (2006). Social choice and the logic of simple games. In Endriss, U. and Lang, J., editors, Proceedings of the 1st International Workshop on Computational Social Choice (COMSOC-2006), pages 125-138. Institute 
for Logic, Language and Computation, Universiteit van Amsterdam, Amsterdam.

Daniëls, T. and Pacuit, E. (2009). A general approach to aggregation problems. Journal of Logic and Computation, 19(3):517-536.

Dietrich, F. and List, C. (2007). Arrow's theorem in judgment aggregation. Social Choice and Welfare, 29(1):19-33.

Dietrich, F. and List, C. (2008). Opinion pooling on general agendas. Working paper, London School of Economics and Political Science. http://franzdietrich.net/Papers/DietrichList-OpinionPooling.pdf.

Dietrich, F. and List, C. (2010). The aggregation of propositional attitudes: Towards a general theory. volume 3 of Oxford Studies in Epistemology, pages 215-234. Oxford University Press, Oxford.

Dietrich, F. and Mongin, P. (2010). The premiss-based approach to judgment aggregation. Journal of Economic Theory, 145(2):562-582.

Fishburn, P. (1970). Arrow's impossibility theorem: concise proof and infinite voters. Journal of Economic Theory, 2(1):103-106.

Herzberg, F. (2010). Judgment aggregators and Boolean algebra homomorphisms. Journal of Mathematical Economics, 46(1):132-140.

Herzberg, F. and Eckert, D. (2012a). Impossibility results for infinite-electorate abstract aggregation rules. Journal of Philosophical Logic, 41(1):273-286.

Herzberg, F. and Eckert, D. (2012b). The model-theoretic approach to aggregation: Impossibility results for finite and infinite electorates. Mathematical Social Sciences, 64(1):41-47.

Herzberg, F., Lauwers, L., Van Liedekerke, L., and Fianu, E. (2010). Addendum to L. Lauwers and L. Van Liedekerke, "Ultraproducts and aggregation". Journal of Mathematical Economics, 46(2):277-278.

Kim, K. and Roush, F. (1980). Introduction to mathematical consensus theory, volume 59 of Lecture Notes in Pure and Applied Mathematics. Marcel Dekker, New York.

Klamler, C. and Eckert, D. (2009). A simple ultrafilter proof for an impossibility theorem in judgment aggregation. Economics Bulletin, 29(1):320-328.

Lauwers, L. and Van Liedekerke, L. (1995). Ultraproducts and aggregation. Journal of Mathematical Economics, 24(3):217-237.

List, C. and Pettit, P. (2002). Aggregating sets of judgments: An impossibility result. Economics and Philosophy, 18:89-110.

List, C. and Puppe, C. (2009). Judgment aggregation: A survey. In Anand, P., Pattanaik, P., and Puppe, C., editors, The Handbook of Rational and Social Choice: An Overview of New Foundations and Applications, pages 457-482. Oxford University Press, Oxford. 
Łukasiewicz, J. and Tarski, A. (1930). Untersuchungen über den Aussagenkalkül. Comptes Rendus des Séances de la Société des Sciences et de Lettres de Varsovie, 23:30-50.

McConway, K. (1981). Marginalization and linear opinion pools. Journal of the American Statistical Association, 76(374):410-414.

Mundici, D. (1986). Interpretation of AF $C^{*}$-algebras in Łukasiewicz sentential calculus. Journal of Functional Analysis, 65(1):15-63.

Pauly, M. and van Hees, M. (2006). Logical constraints on judgement aggregation. Journal of Philosophical Logic, 35(6):569-585.

Rose, A. and Rosser, J. (1958). Fragments of many-valued statement calculi. Transactions of the American Mathematical Society, 87:1-53.

Zlatoš, P. (1981). Two-levelled logic and model theory. In Csakany, B. and Rosenberg, I., editors, Finite algebra and multiple-valued logic (Szeged, 1979), volume 28 of Colloq. Math. Soc. János Bolyai, pages 825-872. NorthHolland, Amsterdam. 